\title{
Aspectus: A Flexible Collaboration Tool for Multimodal and Multiscalar 3D Data Exploitation
}

\author{
DAMIEN VURPILLOT, University of Tours, France \\ QUENTIN VERRIEZ, Bibracte European Archaeological Center, France \\ MATTHIEU THIVET, University of Burgundy Franche-Comté, France
}

\begin{abstract}
High density remote survey technologies have become widespread practices. In recent years, we have seen a tenfold increase in volume of digital data acquired. Consequently, the increasing digitization efforts within the field of archaeology and cultural heritage require new strategies for managing and serving a massive amount of 3D data to a broad array of researchers and an even broader public audience. The Aspectus project aims to facilitate the access to complex three-dimensional data and to promote collaborative work and remote expert assessment. Thus, we can get past the problem of distance and availability of the "object of expertise", ranging from cultural heritage sites to artefacts. By extension, it also enables us to circumvent the ever-present problem of destruction.

In this paper, we give an overview of available applications aimed toward 3D data interaction and their current limitation. We then introduce Aspectus, an ongoing project which takes advantage of available open source solutions to provide a comprehensive application that encompasses multiple complementary approaches: high quality 3D web visualization, data analysis and annotation tools, online workplace platform. We discuss its theoretical framework and its technical implementation. Finally, we present a practical-use case scenario of Aspectus as part of the "Bibracte Numérique" project.
\end{abstract}

Key words:

Collaboration tool, Web-based application, 3D data, Open-source, Remote expert solution.

SDH Reference:

Damien Vurpillot et al. 2018. Aspectus: A flexible collaboration tool for multimodal and multiscalar

3D data exploitation. SDH, 2, 2, 150-165.

DOI : $10.14434 /$ sdh.v2i2.24446

\section{INTRODUCTION}

There is a consensus among cultural heritage and archaeological communities that, in recent years, we have seen a tenfold increase in volume of 3D data acquired. For Richards-Rissetto and von Schwerin [Richards-Rissetto and von Schwerin 2017], we are now collecting an "inordinate amount

\footnotetext{
This work has been developed in the framework of the "Intelligence des Patrimoines" research programme, supported by the Centre-Val de Loire Region and by the Centre d'études supérieures de la Renaissance of Tours.

Author's address: Damien Vurpillot, Centre d'Études Supérieures de la Renaissance, University of Tours, 59 Rue Néricault Destouches, 37013 Tours, France; email: damien.vurpillot@univ-tours.fr; Quentin Verriez, Bibracte European Archaeological Center, Mont Beuvray, Saint-Léger-sous-Beuvray, 71990 Saône-et-Loire, France; Matthieu Thivet, University of Burgundy Franche-Comté, 32 rue Mégevand, 25030 Besançon, France; email: matthieu.thivet@univ-fcomte.fr.

(c) [2018] by the authors; licensee Studies in Digital Heritage, IU, Bloomington (IN), USA. This article is an open access article distributed under the terms and conditions of the Creative Commons Attribution License (CC BY-NC).
} 
of 3D data," while Gordon, Averett and Counts are referring to "archaeological data floods." [Gordon et al. 2016] Digital records and 3D information have been used for a long time in our disciplines, but what was originally circumstantial is now becoming a widespread practice because technical and cost barriers are lowering quickly.

Not only are we facing a new order of magnitude in 3D data recorded, we are also challenged by the exponential complexity of said data. Beyond the increasing density and accuracy, which are already a problem, complexity also arises from a tendency to work with multimodal and multiscalar data sets. Multimodality, from a technical point a view, describes the fact that we are combining multiple means of acquisition and technology: high altitude and low altitude LiDAR (ALS), terrestrial laser scanning (TLS), aerial and terrestrial photogrammetry, structured light scanning (SLS) or even ground-penetrating radar (GPR). Those technologies are not exclusive and prove to be advantageous to employ, depending on circumstances and needs (Table 1).

Table 1. Diagram representing different remote sensing technology for different scales of subject matter.

\begin{tabular}{|c|c|c|c|c|c|}
\hline Landscape & Large site & Medium/Small site & $\begin{array}{c}\text { Construction } \\
\text { Large feature }\end{array}$ & Feature & Object \\
\hline High Altitude ALS & & & & \\
\hline \\
Low Altitude ALS
\end{tabular}

They also provide heterogeneous results with regard to content and scale. Multiscalarity is the property of a system that focuses on the set of interlocking processes that occur at different scales: landscape, site, structure either above or underground, and even objects. Each modality, each individual element, can be useful on its own and often, we are indeed treating them separately. However, the action of combining modalities means to create context, to link data sets together and to enhance our records in order to achieve a better understanding of the study object. In other words, through data fusion [Hess et al. 2015], we are attempting to leverage the inherent multiscalar aspect of multimodal acquisitions to provide context for digital heritage documentation. While the development of comprehensive dynamic digital models is a key component for 3D data analysis, the literature about multi-resolution 3D modeling in archaeology is relatively scarce [Guidi et al. 2009]. This observation may also be related to technological boundaries that have been lifted in recent years. From the data management and data fusion side, photogrammetry and laser scan software are slowly converging toward an all-in-one solution. The visualization and analysis side, which is our focal point of attention, is also evolving very quickly, especially through the release of new web visualizations standards (eg, HTML 5 and WebGL) [Dworak and Kuroczynski 2016] and freely available next generation game engines (eg, Unity or Unreal Engine 4). Online 3D viewers present several advantages. First, they overcome the hindrance problem of specialized software installation, often 


\section{2:152 D. Vurpillot et al.}

proprietary, and file format constraint. By extension, they are also helping with operating platform limitation. Second, they are smoothing user experience by natively streaming data instead of relying on large (and possibly corrupt) archive downloads. Finally, they are extremely flexible in terms of development and debugging.

3D online visualization tools are expected to fulfill multiple needs [Vergnieux and Giligny 2016]. Obviously, displaying 3D objects efficiently is the main purpose. It seems self-evident, but easing access to 3D data is undoubtedly one of the major tasks at hand to overcome the paradox of an increasing amount of complex 3D information, which can be experienced by only a small number of specialists. If we want 3D data to be more than a shiny next generation illustration, often flattened in $2 \mathrm{D}$, and to foster data analysis and interpretation, the sinews of war is data accessibility. A natural extension to visualization is interaction: being able to manipulate data by moving it or moving through it and, to some extent, being able to query information from it. Beyond those basic expectations, one's ability to accurately analyze 3D data is tied to the implementation of generic tools (measurements, profile, etc.) and specialized tools designed with specific research questions in mind. On the other hand, we consider that the interpretative process is closely linked to the ability to "act" over the original 3D data or the analysis results by producing new layers of information, whether it be, for example, surface selection or annotation. This system of layers insures the preservation of original data and analysis and provides common ground for a collaborative workflow. Unfortunately, despite an increasing number of ambitious initiatives towards multi-resolution 3D modeling production [Alexander et al. 2015; Wernke et al. 2015], only a limited number of projects have been able to deploy functional 3D online visualization tools like the MayaArch3D Project [RichardsRissetto and von Schwerin 2017], the Gabii Project [Opitz 2015] or the Via Appia Project [Martinez-Rubi et al. 2016]. Those tools are also custom-developed software, a solution which is only conceivable for a mid-to long-term project with a reasonably sized team of researchers, including developers (professional or non-professional).

For everyone else, other options available are often unsatisfactory or, at least, incomplete [Alliez et al. 2017]. The Ariadne Portal is a great initiative, but its Visual Media Service is only intended to publish visual data as individual "scenes." Inception, another project funded by the European Union [Peter Bonsma et al. 2017], has built up a lot of expectation, but one may be surprised that they still rely on Sketchfab to show off their preliminary results. Sketchfab has indeed been getting a lot of attention lately among other commercial products (Autodesk A360, ShareMY3D, etc.). It is an efficient 3D web viewer, easy to use, which includes the latest web technologies (eg. gltf 2.0 and VR support) and customization options, but again, it is intended to display a single "scene." Open-source self-hosted solutions exist such as Potree for massive point-cloud visualization or 3DHop for mesh display. Cesium is another alternative with a spatial approach, but we will introduce it later, since it is also the base framework of our project. Now, if we move the scope of our investigation toward "interpretation tools," our options are becoming very scarce. The aforementioned software packages allow for basic "highlighting," but advanced editing and annotation are only available through a specific subset of the program. Most of them are desktop products that are no longer being updated (3D Semantic Annotation, ShapeAnnotator, Project Arrigo, Hyper3D, CHER-Ob, etc.). In fact, it seems that Inception and Aioli are now the only robust contenders in this area. Lastly, none of the above 
includes extended collaboration tools similar to booming online workplace platforms such as Slack, Mattermost or Microsoft Team.

\section{THE ASPECTUS PROJECT}

\subsection{Theoretical framework}

Vitruvius introduces the idea of decorum, one of the six principles of architecture [Morgan 1914-1960], by stating that every monument has a meaning and this meaning should arise from its appearance and location (De Architectura, I. 2. 5). Although the extent of the application of Vitruvius's recommendations is open to debate, the idea of aspectus operis, literally the visual impact conveyed by a monument, remains demonstrably practiced throughout antiquity. Therefore, Aspectus was a good fit for our project since our approach is focused on visual representation, a 3D web viewer, but is aimed toward the retrieval of "meaning" and the production of information through the analysis and interpretation of 3D data. The goal of the presented approach to heritage documentation is to propose a flexible web-based team collaboration tool revolving around the interaction with multiresolution 3D spatial data.

The ongoing Aspectus Project [Vurpillot et al. 2017] was born out of necessity a few years ago, during a post-excavation season at Autun (France), while we were working on La Genetoy's monumental district of Augustodunum, civitas capita of the Aedui after they left Bibracte. Needless to say, as an important archaeological site we were running multiple excavations each year, along with extensive survey campaigns ranging from long-range to close-range remote sensing. Our first intent was to fuse multi-resolution 3D data coming from high altitude LiDAR surveys, geophysical surveys and many photogrammetrical surveys spread over multiple excavations and recorded iteratively at diverse degrees of progress. At first, our goal was only to deliver a comprehensive 3D representation of the monumental district's archaeological activity (Fig. 1), that is, a broad overview available online for researchers and the general public. Soon enough, we were also required to produce a 3D reassembly from fragmented artefacts, to perform surface analysis over them, to share the results and discuss the results. Our simple web viewer was obviously not suitable for this approach. It became very clear to our research team that to absorb such heterogeneous data (scale, angle of research), developing a specific program was now a necessity, since none of the available solutions included the comprehensive toolset that we needed.

Early in the development process, we set cornerstones for the project:

1. Ease of access is our main concern. Since archaeology is "going digital" and 3D data is everywhere, we had to make a leap forward and make sure that everyone involved in our research team should be able to access this information, regardless of the platform he is working on or his background in information technology. For that reason, we chose to develop a web platform, also taking into account the other benefits presented by this technology. By extension, such a tool is only appealing if it proves easy to use and builds bridges with older methods and work habits. 


\section{2:154 D. Vurpillot et al.}

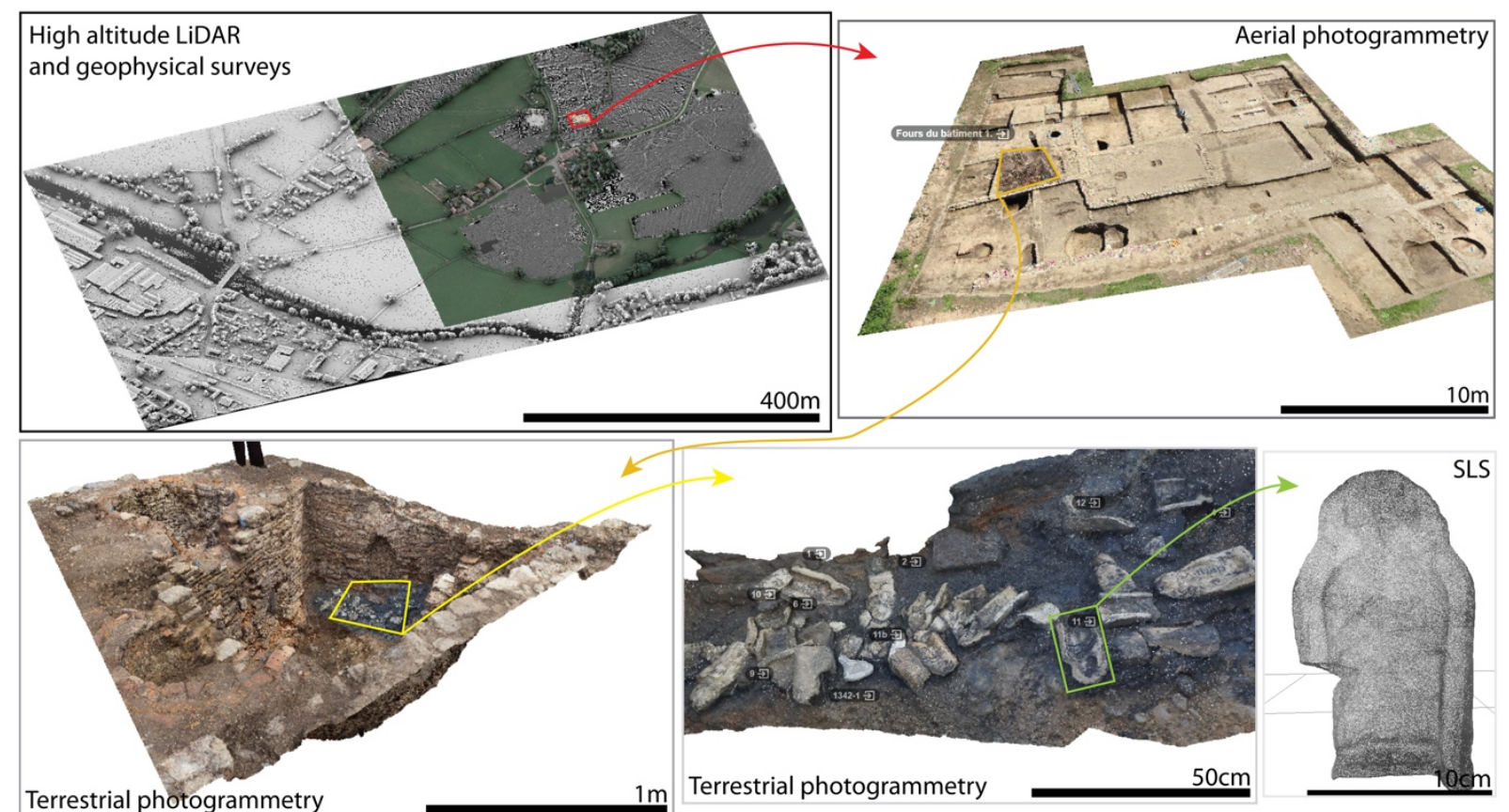

Figure 1. Screen excerpts from our multi-resolution 3D web-viewer for the Augustodunum Project at various scales.

2. Preservation of the information context is also another concern. One idea we have developed is the production of a project-wise dynamic digital model that is able to evolve. We can easily draw an analogy with the so called "Russian doll" where each 3D data set is encapsulated in a wider data set, but thanks to the hierarchical level of details applied to multimodal data integration, we are able to maintain full resolution of each entire data set. Since data fusion happens through spatial relationships, we are also able to enforce model flexibility by adding or removing the data set over time. A comprehensive approach also has its downsides, and one can easily get overwhelmed by the amount of information. In this regard, we developed what we call "isolation pods", which describe the ability to explore visually any subset of data outside its spatial context while maintaining a symbolic link; thus, one is always able to move backward or forward to any related context. This kind of relative disconnect is also practical for introducing elements for which the spatial information is fuzzy or absent.

3. Consequently, spatial references are a crucial aspect of our data sets. At best, it means that we also have the opportunity to supplement 3D information with GIS data. At worst, if spatial references are almost systematically recorded as part of archaeological activities, it may not be a widespread practice in other area of cultural heritage. With such a dense weave and weft of interlacing heterogenous data, we are getting close the philosophy of a Building Information Model (BIM) or HBIM for cultural heritage management [Barazzetti et al. 2016]. A promise of cloud-BIM is to produce a centralized version of a model that can be remotely 
accessed by multiple specialists, who can explore their share of data while keeping in sight other information and activities happening in a virtual surrounding.

4. Collaboration is indeed one of our primary concerns. By broadening the availability of 3D data, we were also hoping to foster teamwork and cooperation. Engaging in this endeavor requires us to put forth strong efforts toward ease of access, as we said earlier, because collaboration rests on the user's willingness to adopt new tools and good rates of voluntary adhesion to what is essentially a professional social network. Moreover, acting over 3D data is made possible through generic and specialized tools, but one also needs a means of communication, like cloud-annotation and chatroom, to convey analysis and interpretations to other network members.

5. Lastly, flexibility was deemed necessary in order for Aspectus to be transposable to other projects. Our development approach is aimed toward progressive enhancement. We established a core set? of minimal social functionality as a "collaborative layer" acting over 3D web viewer's instances featured with? a generic toolset. More advanced functionalities are built as needed depending on the project and with specific research questions in mind. Once we have reached a user experience that fits the needs of the project from a research point of view, we are applying "graceful degradation" to serve different user groups: project manager, active and guest researchers, general public, etc. Functionalities are progressively reduced to meet the expectations of each user group and to adjust within each use-case? scenario. By extension, our efforts were also directed toward interoperability in order to ease the process of integration within existing workflow frameworks. In our case, we were interested in linking Aspectus with an archaeological field data collection and management system.

\subsection{Technological insights}

Aspectus is an ongoing project at an early stage of development. We divided the workload into three main tasks: data management (A), data visualization (B), and data enrichment (C). Currently, we are putting forth strong efforts toward data visualization, since dynamic display of multi-resolution 3D spatial data is one of our core features. Subsequently, our technological choices will lay the groundwork for other development aspects. In this regard, we agreed as a team that it would be more profitable to rely on existing open-source solutions that are prone to enhancement and personalization, rather than to reinvent the wheel. There are multiple reasons behind this choice: we are not professional developers and we do not have the means to dedicate ourselves to full-time development. Moreover, there are plenty of available open-source solutions that one can link together and leverage as blocks to achieve a more comprehensive platform. By enhancing those blocks and carefully weaving links between them, we are fast-tracking the development process. Our main visualization block is Cesium, a JavaScript library for 3D geospatial visualization [Pinkos 2017]. It uses WebGL and is cross-platform, cross-browser, and tuned for dynamic-data visualization. We will not dive into the depth of HTML5 and WebGL [Dworak and Kuroczynski 2016] but the ability to provide hardware supported rendering using a graphics card (GPU) through a browser has been a game changer. Cesium is extremely interesting because it natively displays a wide variety of spatial information with dynamic capabilities (Fig. 2). Additionally, because Cesium was developed with 


\section{2:156 D. Vurpillot et al.}

massive heterogeneous data display in mind, a lot of attention has been paid to optimization, especially with the implementation of multiple level of details (LOD) algorithms (Discrete LOD, Continuous LOD, Hierarchical LOD) ruled by a Screen-Space Error mechanism [Cozzi and Ring 2011]. Furthermore, this library is under active development and benefits from a growing community.

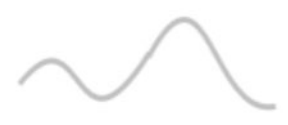

Raw Terrain DEM
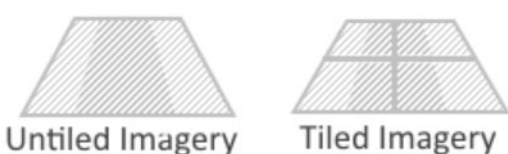

Tiled Imagery

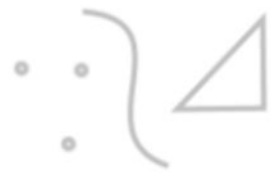

Traditional

Vector Formats

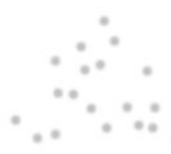

Raw Point Clouds

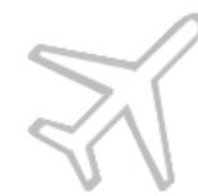

Web-Unfriendly 3D Models

Figure 2. Depiction of the wide variety of spatial information natively supported by Cesium.

In Aspectus, a first instance of Cesium, called "the hub", behaves as the entry point of our 3D visualization. Spatial data sets are then displayed on top of a 3D globe. Depending on the project, the density of a 3D object and all related information may vary and can be passively filtered using LOD algorithms by moving the camera directly to point of interest and actively filtered by interacting with the menu. The hub's purpose is really to provide a broad overview of available data sets for any given project along with some information accessible by clicking on a 3D object. That information can be hard-coded directly inside the data (derived from native file attributes, for example) or linked to a database. At the moment, it is not intended to be able to run cross data analysis over massive 3D spatial data sets directly into "the hub". Beside the sheer amount of computing power obviously required, we feel that it is the kind of task that should be operated outside of Aspectus and with specialized software. Furthermore, in order to preserve navigation efficiency and quality, we intend to provide tools with only a light impact over performances. Otherwise, we would go against the general philosophy of cross platform compatibility and ease of access. It is also a reminder that "graceful degradation" is tuned in the direction of user groups with specific needs and should not be limited by one's platform.

From "the hub", one can then move to a second instance of 3D visualization, what we call "isolation pods". Even if a point of interest (a site, a structure, etc.) is visible and can be queried via "the hub", it is our understanding that working on a subset of data, isolated from its global visual context, appears to be more practical. We are allowing two different isolation modes. First, one is another instance of Cesium aimed toward visual fidelity, by means of high resolution meshes optimized for web display (glTF 2.0 with hierarchical LOD), and temporal visualization for which a slider is available and allows users to observe data evolution over time (for example, excavation progress). Second, one is built from Potree, another JavaScript library for massive point cloud visualization, and is subtitled "metrological instance". The subtlety at play here is that we are now only working with point clouds. As a raw byproduct of any 3D acquisition, point clouds are as accurate as the acquisition itself, but the process of 3D surface reconstruction is inherently degrading spatial information by skipping points or by creating intermediary points as a/the vertex: "The goal of surface reconstruction can be stated as follows: given a set of sample points P assumed to lie on or near an unknown surface S, create a surface model $S^{\prime}$ approximating $S$. A surface reconstruction procedure cannot guarantee the 
recovering of S exactly" [Remondino 2003]. Most of the generic tools, which include measurement tools, are available through the "metrological instance".

Most data-management requirements are inherited from the joint use of Cesium and Potree. Vectors are formatted as JSON or directly as CZML, which is a specific variation of JSON standard. Imagery, either tiled (WMS, TMS, etc.) or untiled, is usually served as PNG. Point clouds can undergo a different type of conversion and indexing depending on the situation. For small to medium data sets, LAZ format [Isenburg 2013] will be preferred for Potree, whereas PNT format [Lilley et al. 2017], based on JSON standard, will be used with Cesium. For massive data sets, both Cesium and Potree require a specific indexing, namely entwine [Manning 2017], with help from an auxiliary streaming server named Greyhound. Finally, complex 3D models are served as glTF 2.0. The glTF standard is promoted as the "JPEG of 3D" by the Consortium Khronos Group (including Intel, NVIDIA, Google, Sony, Apple, Samsung, IBM). A glTF asset is constituted by a JSON file (node hierarchy, cameras, materials), binary files (geometry, animation, etc.) and JPG or PNG files [Trevett 2017]. From a back-end perspective, we are willingly pushing open-source format, but the amount of work dedicated to achieve that goal can vary extensively, depending on the existing workflow and data-management behind different projects. More often than not, it can prove to be a bit chaotic in archaeology. We are also suggesting the use of PostgreSQL databases with a PostGIS extension. Some people are also looking into NoSQL databases such as Apache Couch, but it seems that spatial index extensions like Geocouch are not advisable yet in terms of performance.

Finally, data enrichment includes team collaboration, tools and services. Our intention is to smoothly compartmentalize data visualization and data enrichment so that the latter can be displayed as an overlay. Implementation of this asynchronous mechanism will be imperceptible for the user. We are aiming for stability on the data visualization side with a limited number of active curators for each project. Users will be able to act on available data sets but not to directly modify any original data. Information produced by users will be displayed as a new layer on top of the original data, and since they will be available for export as JSON, it is up to project managers and data curators to decide if they want that new data to be injected into the original data. If not, information will remain on the user side and will still be available to be shared with other users. We are also leveraging the inherent advantage of working with spatial data, since what is produced in our metrological instance will be automatically available on other instance pods because every instance shares a common spatial context. Aspectus' collaborative platform is based on Riot.IM, an open source and self-hosted solution which is cross-platform and cross-browser. It is also built on top of the Matrix open standard, which is natively making a very strong attempt to bridge to as many different networks as they can. There are already hosted bridges to Slack, Gitter, several IRC networks and even Twitter or SMS, and bridges you can host yourself for Telegram or XMPP. On the security side, Matrix has implemented decentralized End-to-end Encryption (e2e), allowing information to be encrypted by the sender in such a way that only the people you are communicating with can read it but none of the servers in between can decrypt the information [Hodgson 2017]. That side of the Aspectus project is still at an early stage of development, but we are confident that we were able to gain a broad overview of the philosophy behind the project and how we intend to implement it from a technical point of view (Fig. 3). 


\section{2:158 D. Vurpillot et al.}

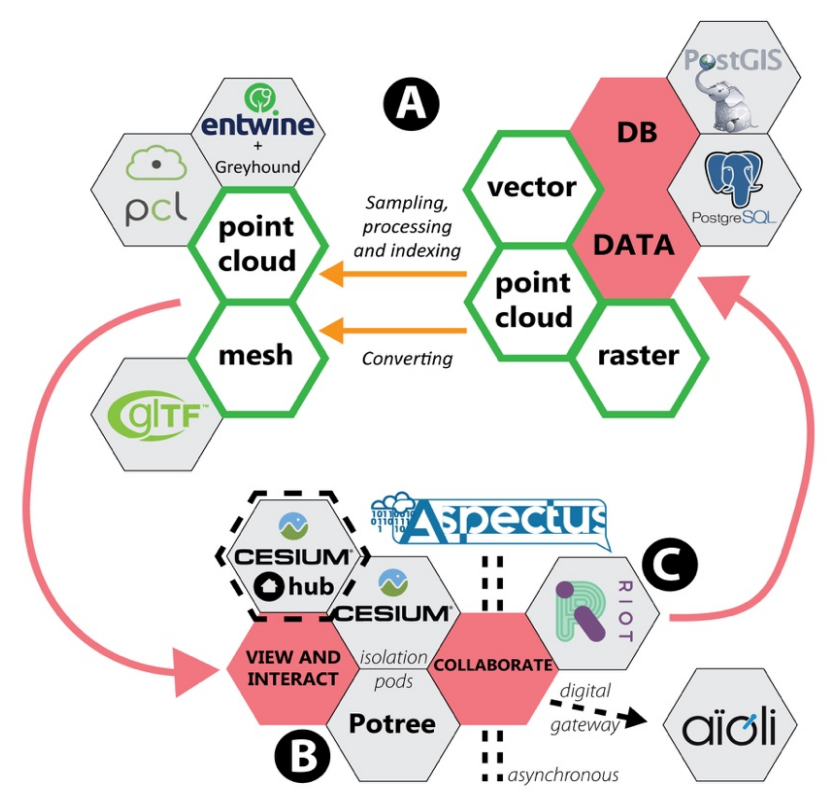

Figure 3. Schematic depiction of the Aspectus project theoretical framework with a) data management, b) data visualization and c) data enrichment.

\section{CASE STUDY: BIBRACTE}

\subsection{Bibracte Numérique and Bibracte PC2 excavation}

The oppidum of Bibracte, capital of the Aedui in the first century BC, is located at the top of Mont Beuvray in Burgundy. For more than 30 years, it has been associated with a European Archeological Center that includes an archaeological site (awarded status of "Grand Site de France"), a museum (awarded status of "Musée de France") and a research center hosting multiple international teams every year. Explored since the end of the 19th century, Bibracte is now distinguishing itself by an extensive production of high-quality archaeological data, and it is hosting a wide variety of researchers and specialists. Such concentration of archaeological data, produced by means of numerous scientific, cultural and tourist missions, makes Bibracte a rich and complex place where many actors from the European archaeological community gather and exchange ideas. For the past few years, the amount of digital data produced annually has become so extensive that the research center's capacity to manage and exploit this information influx is dangerously close to becoming congested, impacting, as a consequence, the pace of scientific publication. This situation illustrates clearly some issues and concerns expressed in the introduction. Consequently, in the course of 2017, Bibracte engaged a new program for digital equipment and digital tools development: Bibracte numérique (BibNum). BibNum is aimed toward a wide scope of users. Indeed, professional archaeologists are concerned but also a broad variety of specialists and researchers linked to ongoing projects, students, and, more subtly, the general public. Synergy between BibNum and Aspectus arises from the joint philosophy of both projects: in light of an integrated approach, we are 
focusing on experimentation and experience sharing with every group of people involved in Bibracte research programs. It was only natural that we tried to integrate Aspectus to a broader BibNum initiative. This program is conducted in consultation with various archaeological community representatives gathered in a steering committee (Aoroc, ArScan, Bibracte EPCC, MOM J. Pouilloux, MSHE C.-N. Ledoux, Ministry of Culture and Communication - Sous-direction de l'archéologie and INRAP). More specifically, the project aims to develop a digital archaeological ecosystem and to provide testing grounds for learning and experimenting with new digital practices related to the various activities covered by the center (not only archeology but also museography, education and tourism). Thereby, we expect to facilitate interaction between actors, not only within Bibracte but also from outside, and to foster communication, exchange and transmission of knowledge.

In this ambitious project, BibNum is divided into four reflection groups:

1. Dissemination of knowledge through archaeological mediation based on the potential of digital technology;

2. Development of digital equipment (hardware and software) for selected excavations to support digital field recording and foster on-site interaction with visitors;

3. Implementation of an innovative archaeological knowledge production process based on the potential of digital data and organization of the above-mentioned knowledge for dissemination toward different audiences;

4. Implementation of appropriate digital infrastructures.

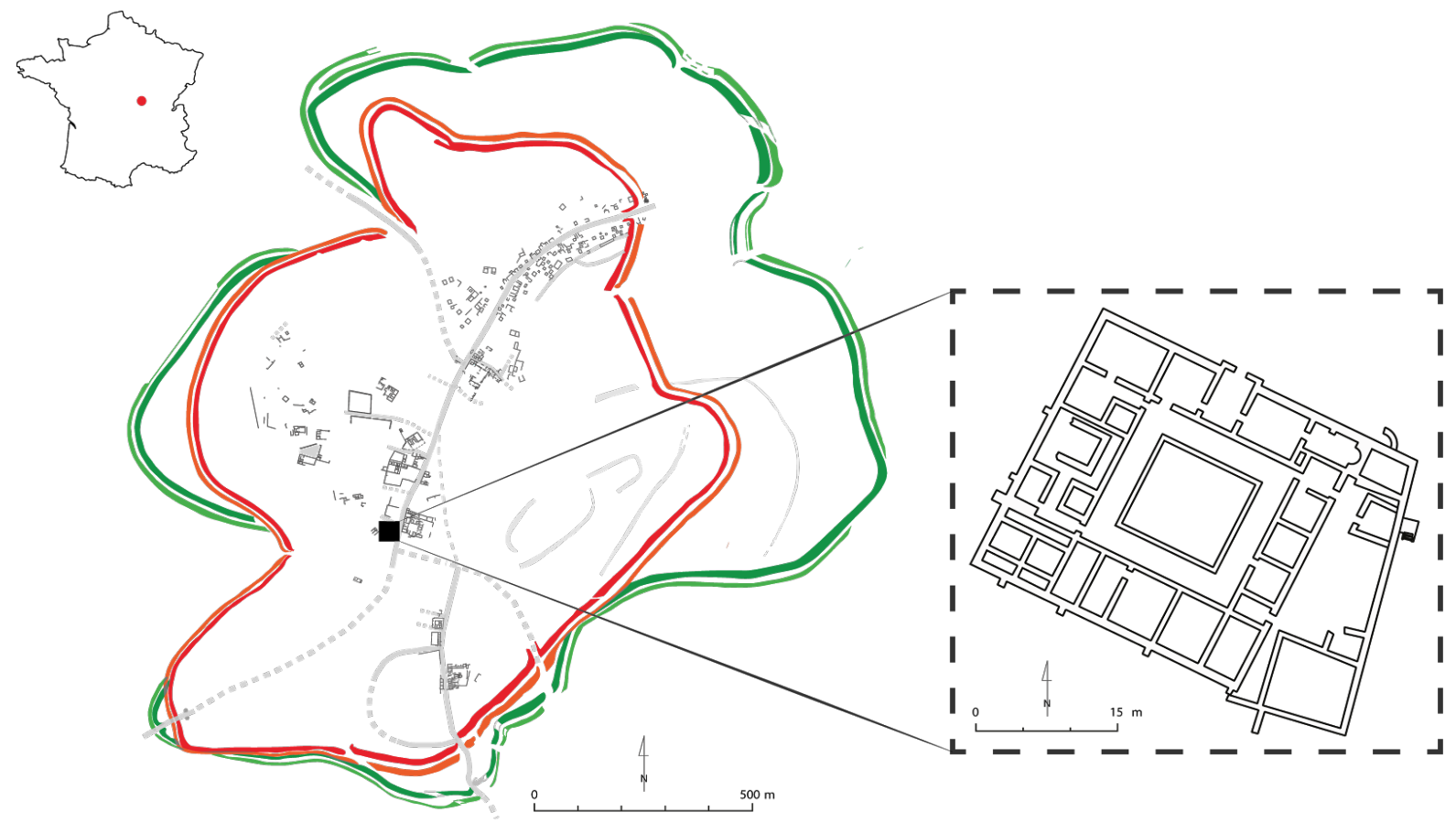

Figure 4. Geographical localization of the oppidum of Bibracte and location of the domus PC2 within the city wall. 
We selected the PC2 excavation of a gallo-roman domus as a pilot site (Fig. 4). It was also decided that development and deployment of on-site digital equipment should be implemented and tested progressively throughout the fieldwork. The building itself is a $1500 \mathrm{~m}^{2}$ characteristic luxurious Roman urban house located at the heart of the Gallic agglomeration and dated to the latest settlement phase of the site (around $30 \mathrm{BC}$ ). Unearthed vestiges are particularly well preserved. In some places, we can observe up to four meters of masonry. Every year, we gather an excavation team made up only of teenagers who have come to learn the basics of archeology under the supervision of professionals. In that respect, due to its nature as a school-site, the PC2 excavation must be extremely didactic and maintain exemplary field recording. The quality of preserved remains associated with the slow and meticulous excavation of PC2 provides a perfect case study for the establishment of a "connected archaeological site", with the opportunity to experiment with new protocols and technologies in situ, for example, the improvement of 3D acquisitions. In 2017, the excavations were focused on the north-eastern quarter of the building, covering approximately $400 \mathrm{~m}^{2}$. In this first season of excavation, we experimented with an innovative approach directly related to Aspectus: systematic photogrammetry. Thus, at each step of the excavation, we tried to obtain a comprehensive digital recording of each archaeological layer unearthed. Our equipment was selected according to the size of the area to be recorded: a digital reflex camera for smaller areas, the same device on a pole with remote shutter release for larger surfaces, and a drone for site-wide acquisitions (Table 2).

Table 2. Specifications of the cameras used for photogrammetry on PC2 excavation in 2017.

\begin{tabular}{|c|c|c|}
\hline & Camera & Drone \\
\hline Camera & Canon EOS 6D & DJI Phantom 4 pro \\
\hline Sensor size & Full Frame (24 x 36 mm) & $1^{\prime \prime}(8.8 \times 13.2 \mathrm{~mm})$ \\
\hline Effective pixels & $20.2 \mathrm{M}$ & $20 \mathrm{M}$ \\
\hline Resolution & $5472 \times 3648$ & $5472 \times 3648$ \\
\hline Focal & $35 \mathrm{~mm}$ & $9 \mathrm{~mm}(35 \mathrm{~mm}$ equivalent $=24 \mathrm{~mm})$ \\
\hline Aperture range & $f / 3.5-f / 4.5$ & $\mathrm{f} / 4.5-\mathrm{f} / 7.1$ \\
\hline Shutter range & $1 / 60-1 / 150$ & $1 / 160-1 / 200$ \\
\hline ISO range & $200-400$ & $200-400$ \\
\hline File format export & JPEG - DNG (RAW) & JPEG - DNG (RAW) \\
\hline
\end{tabular}

In regard to the overlap required between images, our approach was empirical with the reflex camera (around 70\% overlap), while drone flights were automatically set up via a proprietary application called DJI GS PRO and also retained around 70\% of overlap. The photogrammetric processing of digital images was performed with Agisoft PhotoScan 1.2 with the aim of maintaining, as much as possible, a $1 \mathrm{~mm}$ /pixel resolution for orthophotography and 3D model production. In the end, the 
entire dataset included 42 acquisitions for a total of 92 Gigabyte of data (images, processing and results). This raw data provided a complete and representative corpus of an archaeological excavation campaign, ideal for developing a first test with Aspectus.

\subsection{Musings on future directions}

While the PC2 2017 case study was compelling, Aspectus was only implemented for testing. Our goals were first to evaluate the robustness of our 3D visualization system and second to assess the workload required to process a great variety of heterogeneous data sets that were not optimized at all to fit in our pipeline. We had to bring together some of the previous recording (raster maps, various GIS datasets and LiDAR) with the new comprehensive photogrammetric recording of PC2. The "hub" gave access to a multi-resolution terrain model derived from LiDAR data with an overlay of highresolution satellite imagery or a hillshade raster. On top of it, we displayed multiple JSON (past excavation, recent excavation and interpretation) extruded in 3D with contextual information and multi-resolution mesh of our PC2 excavation (Fig. 6). From here, it was possible to access the so called "isolation pods" related to PC2: one Cesium instance with the same multi-resolution mesh and temporal information from the excavation where each step is visible and queryable; one Potree instance where each step of the excavation can be visualized as point clouds with a comprehensive tool set for metrological inspection.

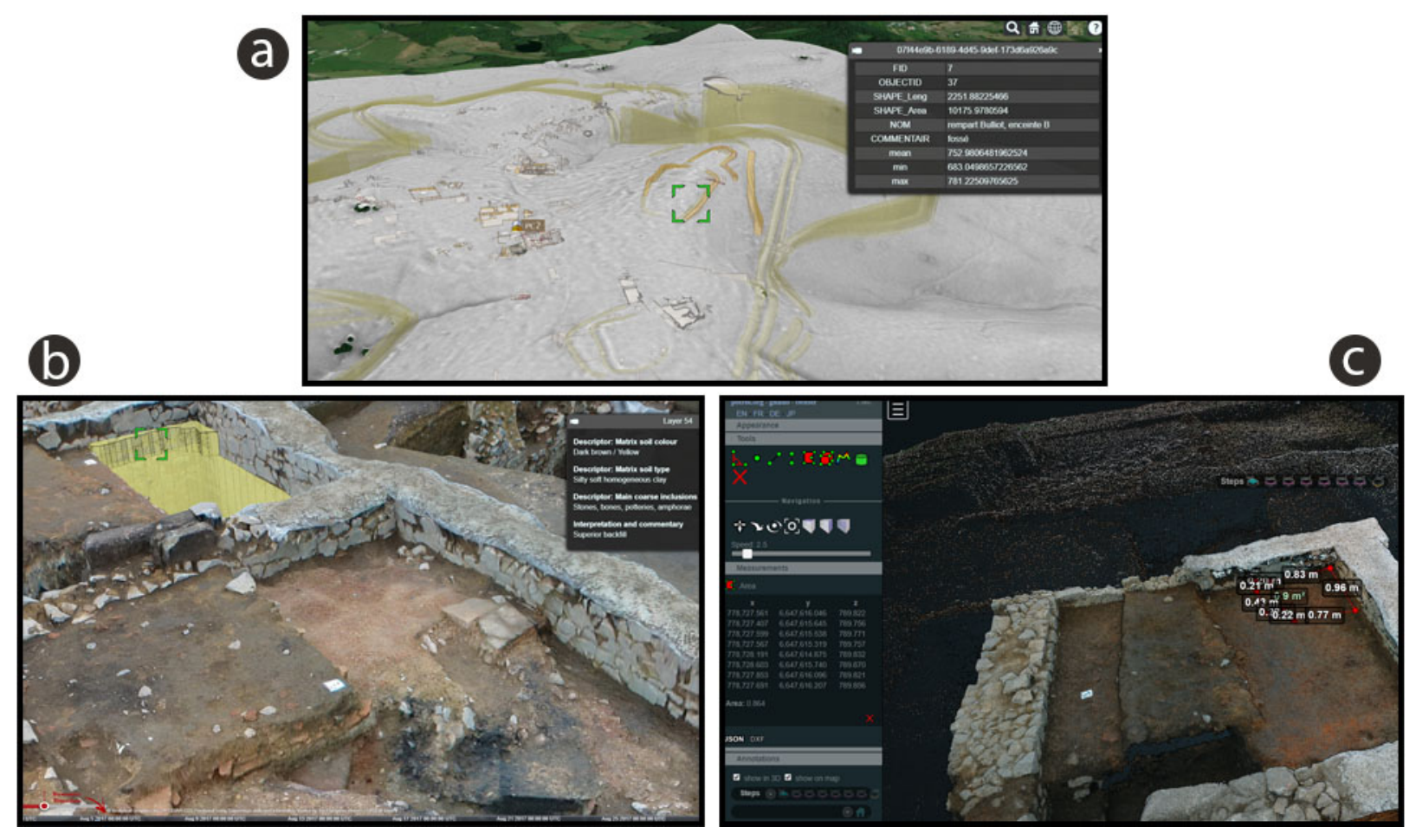

Figure 6. Aspectus applied to Bibracte with a) "The Hub" b) Cesium instance pod and c) Potree metrological instance. 


\section{2:162 D. Vurpillot et al.}

Moving forward, since BibNum is also an ongoing project at an early stage of development, the definition of a precise workflow and its technical implications are still under discussion. Regardless, it proved to be a rewarding experiment for both projects. We were able to assess the current situation of data management and to explore possible enhancement. Unexpectedly, the most significant hindrance originated from ordinary spatial data, most of them being older shapefiles. Moving from a "dirty" shapefile to a "clean" JSON can prove really time consuming. By "clean", we mean retaining a clear and efficient structure for storing geometry, appearance and attributes in accordance with the JSON standard. Attributes were in this case hardcoded inside the data itself, since no global database was operational at the moment. As a side note, shapefiles with z-values were difficult to manage because it seems that Agisoft Photoscan is unable to close geometry when people are directly drawing over photogrammetry. It must also be noted that the pipeline (Cesium Terrain Builder) to produce a high-quality multi-resolution terrain model from a digital elevation model (DEM), in our case LiDAR data, is challenging, to say the least. Native 3D information data sets, in the form of point clouds, were clearly easier to interact with, both in terms of initial processing and multi-resolution mesh generation. Undoubtedly, it was related to the fact that most of the 3D acquisitions were made recently and produced with anticipation about current data-management challenges. We were expecting such disequilibrium between optimized and unoptimized data sets, but the order of magnitude was outstanding in comparison with our initial assessment. Moreover, a performance audit carried out with Chrome development tools shows clearly that dense layers of spatial data displayed in 3D have a massive impact on load time if we rely on non-minified JSON and extrusion only. For example, we can expect considerable improvement by directly converting that spatial information as 3DTiles. From a broad perspective, we wish to add a systematic optimization step at the data-management stage to reduce file size and, consequently, load time. Besides, there is still lot of room for improvement on the script side. We are also looking forward to strengthening synergy between different software blocks. For instance, Potree main developer M. Schuetz is currently trying to allow Potree to be displayed on top of Cesium. This could prove to be a major enhancement, since point cloud display with 3DTiles is now relatively limited both in terms of performance and customization. Potree is also convincing when it comes to drawing in 3D over existing point cloud data. That new layer of user-made data, produced with Potree and stored in JSON, will then be available for display either in Potree or Cesium. It has become clear to us that JSON should be used directly in Cesium for small datasets, like user "drawing", but they must systematically be converted as 3DTiles as soon as they get more sizeable.

\section{CLOSING THOUGHTS}

The increasing digitization efforts within the field of archaeology, and more generally cultural heritage, requires strategies for managing and serving an inordinate amount of 3D data to a broad array of researchers and an even broader public audience. At present, we are facing a vastly compartmentalized market and we recognize the lack of a comprehensive software solution that encompass multiple complementary approaches: high quality 3D web visualization, data analysis and annotation tools, and an online workplace platform. In other words, making complex 3D data accessible and prone to analysis in a collaborative environment. We are trying to close this gap with Aspectus but we are still at an early stage of development. A robust theoretical framework has been

Studies in Digital Heritage, Vol. 2, No. 2, Publication date: December 2018 
set with multiple cornerstones in mind: ease of access and collaboration incentives, flexibility, and preservation of the information context through the use of spatial data. Most of our efforts were directed toward the latter. What blocks do we choose to efficiently display the information and how do we manage the information that we feed to those blocks Both Augustodunum and Bibracte were proving grounds in this regard. Cesium and Potree proved to have great synergy and to be complementary in terms of visualization and interactions. They also provide great flexibility for a project with such a small team of archaeologists behind it and almost no funding. This is also why the former cornerstone, collaboration, is still lagging behind. Therefore, we are relying on partnership to move forward and, recently, we have been discussing the possibility of creating a bridge with the above-mentioned Aioli project. They would benefit from contextualization and spatialized information whereas we would learn a lot from their multi-layer data annotation tool. Moreover, we are also both looking for the implementation of a joint digital collaboration platform. Finally, today there are other praiseworthy initiatives seeking in different ways to engage the issue of serving an inordinate amount of 3D data, like the Inception project. We are working at a very different level: they are funded by the European Union and have a considerable team of researchers available; they are also already exploring a complex semantic ontology approach for HBIM. Ontologies are a core feature of the Semantic Web imitative and are essential for enhancing communication between archaeologists and, moreover, between archaeologists and people in other knowledge fields. Thus, the implementation of such knowledge representation is set to be the next milestone in Aspectus' development.

\section{ACKNOWLEDGEMENTS}

The authors gratefully acknowledge the support of MSHE C. N. Ledoux, Bibracte European Archaeological Center and the University of Burgundy Franche-Comté.

\section{REFERENCES}

Craig Alexander, Axel Pinz, and Christian Reinbacher. 2015. Multiscale 3D rock-art recording. Digital Applications in Archaeology and Cultural Heritage, 2 (2), 181-195.

Pierre Alliez, Laurent Bergerot, Jean-Francois Bernard, et al. 2017. Digital 3D Objects in Art and Humanities: challenges of creation, interoperability and preservation. White paper.

Luigi Barazzetti, Fabrizio Banfi and Raffaella Brumana. 2016. Historic BIM in the Cloud. In Marinos Ioannides, Eleanor Fink, Antonia Moropoulou, et al., eds. Digital Heritage. Progress in Cultural Heritage: Documentation, Preservation, and Protection. Proceedings of the 6th International Conference, EuroMed 2016, Nicosia, Cyprus, October 31 - November 5, 2016, Proceedings, Part I. Springer International Publishing, 104-115.

Patrick Cozzi and Kevin Ring. 2011. 3D engine design for virtual globes. CRC Press.

Peter Bonsma, Iveta Bonsma, Anna Elisabetta Ziri et al. 2016. INCEPTION Standard for Heritage BIM Models. In Marinos Ioannides, Eleanor Fink, Antonia Moropoulou, et al., eds. Digital Heritage. Progress in Cultural Heritage: Documentation, Preservation, and Protection. Proceedings of the 6th International Conference, EuroMed 2016, Nicosia, Cyprus, October 31 - November 5, 2016, Proceedings, Part II. Springer International Publishing, 590-599. 


\section{2:164 D. Vurpillot et al.}

Daniel Dworak and Piotr Kuroczyski. 2016. Virtual Reconstruction 3.0: New Approach of Web-based Visualisation and Documentation of Lost Cultural Heritage. In Marinos Ioannides, Eleanor Fink, Antonia Moropoulou, et al., eds. Digital Heritage. Progress in Cultural Heritage: Documentation, Preservation, and Protection. Proceedings of the 6th International Conference, EuroMed 2016, Nicosia, Cyprus, October 31 - November 5, 2016, Proceedings, Part I. Springer International Publishing, 292-306.

Jody Gordon, Erin W. Averett, and Derek Counts. 2016. 0.2. Mobile Computing in Archaeology: Exploring and Interpreting Current Practices. In Jody Gordon, Erin W. Averett, and Derek Counts, eds. Mobilizing the past for a digital future: the potential of digital archaeology. The Digital Press, The University of North Dakota, 1-30.

Gabriele Guidi, Michele Russo, Sebastiano Ercoli, et al. 2009. A multi-resolution methodology for the 3D modeling of large and complex archeological areas. International Journal of Architectural Computing, 7(1), 39-55.

Michael Hess, Vid Petrovic, Dominique Meyer, et al. 2015. Fusion of multimodal three-dimensional data for comprehensive digital documentation of cultural heritage sites. In Proceedings of Digital Heritage - International Congress 2015, 2(2), IEEE, 595-602.

Matthew Hodgson. 2017. Encrypting Matrix Building a universal end-to-end encrypted communication ecosystem with Matrix and Olm. (FOSDEM 2017). Retrieved January 05, 2018 from https://matrix.org/blog/wp-content/uploads/2017/02/2017-02-03.1-FOSDEM-EncryptingMatrix.pdf

Martin Isenburg. 2013. Laszip. Photogrammetric Engineering \& Remote Sensing, 79(2), 209-217.

Sean Lilley, Tom Fili, Patrick Cozzi and Dan Bagnell. 2017. 3D-Tiles/PointCloud. (2017). Retrieved January 04, 2018 from https://github.com/AnalyticalGraphicsInc/3dtiles/tree/master/TileFormats/PointCloud

Connord Manning. 2017. Entwine. Trillions of points. Massive point clouds as infrastructure. (2017) Retrieved January 04, 2018 from https://entwine.io/slides/foss4g2017/index.html\#/

Oscar Martinez-Rubi, Maurice de Kleijn, Stefan Verhoeven, et al. 2016. Using modular 3D digital earth applications based on point clouds for the study of complex sites, International Journal of Digital Earth, 9:12, 1135-1152, DOI:10.1080/17538947.2016.1205673.

Morris Hicky Morgan. 1914-1960. Vitruvius: The Ten Books on Architecture. Harvard University Press.

Rachel Opitz. 2015. Teaching Practice while Developing Practice: Mobile Computing at the Gabii Project Field school. Paper read at the Mobilizing the Past for a Digital Future: The Potential of Digital Archaeology Workshop, 28 February 2015, Boston.

Hannah Pinkos. 2017. Why 3D? The benefits of 3d geospatial visualization beyond pretty pictures. (FOSS4G 2017). Retrieved January 02, 2018 from https://cesium.com/presentations/files/why3d.pdf

Fabio Remondino. 2003. From point cloud to surface. International Archives of photogrammetry, Remote Sensing and spatial information sciences, 34. Swiss Federal Institute of Technology Zurich (Institute of Geodesy and Photogrammetry).

Heather Richards-Rissetto and Jennifer von Schwerin. 2017. A catch 22 of 3D data sustainability: Lessons in 3D archaeological data management \& accessibility. Digital Applications in Archaeology and Cultural Heritage. 6, 38-48. DOI: https://doi.org/10.1016/j.daach.2017.04.005 
Neil Trevett. 2017. glTF 2.0 Launch. Web3D Conference. (June 2017) Retrieved January 04, 2018 from https://www.khronos.org/assets/uploads/developers/library/2017-web3d/glTF-2.0Launch_Jun17.pdf

Robert Vergnieux and François Giligny. 2016. Pour un usage raisonné de la 3D en archéologie. In Robert Vergnieux and François Giligny, eds. Les Nouvelles de l'archéologie, 146. Éditions de la Maison des sciences de l'homme, 3-5.

Damien Vurpillot, Quentin Verriez and Matthieu Thivet. 2017. Aspectus: Plateforme collaborative en ligne d'analyse des données 3D pour l'archéologie et le patrimoine. Retrieved January 25, 2018 from https://aspectus.hypotheses.org/

Steven Wernke, Gabriela Oré, Carla Hernández et al. 2016. 2.3 Beyond the Basemap: Multiscalar Survey through Aerial Photogrammetry in the Andes. In Jody Gordon, Erin W. Averett, and Derek Counts, eds. Mobilizing the past for a digital future: the potential of digital archaeology. The Digital Press, The University of North Dakota, 251-278.

Received September 2018; revised October 2018; accepted December 2018. 